\section{Posibles criterios clínicos-epidemiológicos para el diagnóstico de loxoscelismo}

\section{Possible clinical-epidemiological criteria for the diagnosis of loxoscelism cutaneous and visceral cutaneous}

\section{Sr. Editor:}

Entre los problemas médicos causados por los artrópodos, el aracnoidismo y específicamente el loxoscelismo es uno de los más importantes. El género Loxosceles tiene alrededor de 70 especies, con variaciones según la región. En Latinoamérica predomina la especie Loxosceles laeta ${ }^{1}$. Produce principalmente dos cuadros clínicos definidos, el cutáneo-necrótico y el cutáneovisceral, con diferente pronóstico y manejo médico ${ }^{2}$. Estudios han abordado ciertas variables del problema, como el realizado por Sanabria y cols. ${ }^{3}$, que da cuenta del panorama epidemiológico del loxoscelismo en Perú. En este estudio se encontró un mayor reporte de mordeduras en la zona costera del Pacífico, aumentando los casos en verano y disminuyendo en invierno. Los accidentes ocurrieron más frecuentemente en horas de la noche y en la mañana. A su vez en Chile, Ríos y cols. ${ }^{4}$, en un estudio basado en consultas telefónicas del Centro de Información Toxicológica de la Universidad Católica de Chile (CITUC), encontraron resultados similares. En el año 2003, Valverde ${ }^{5}$ determinó las características clínico-epidemiológicas del loxoscelismo en un hospital de Perú. En este estudio, las amas de casa fue el grupo más afectado, la mayoría perteneciente a zonas urbanas y urbano-marginales, con una mayor frecuencia en los meses de verano y otoño. El dato clínico más común fue el dolor de tipo urente $(100 \%)$ y en $55 \%$ de los casos no se identificó la araña. Webb y cols. ${ }^{6}$ encontraron como factores asociados a la presentación de loxoscelismo cutáneo-visceral, la fiebre, el compromiso del estado general y la mordedura en el tórax. A pesar de toda esta evidencia, en las guías de manejo de loxoscelismo, no se emplean las variables estudiadas para el diagnóstico ${ }^{7-9}$. La identificación del arácnido sigue siendo el primer paso en el algoritmo diagnóstico, aún cuando en más de $50 \%$ de los casos esto no es posible. Ante lo mencionado, variables como el género, lugar del accidente, ruralidad, hora del día, estación del año, ocupación, tiempo de enfermedad, signos y síntomas (dolor tipo urente, edema, eritema, fiebre, malestar general, necrosis), lugar de la lesión (extremidades), características de la lesión (placa livedoide, eritema, presencia de ampollas o flictenas, bor- des necrosados o signos de necrosis, signo de mordedura) y su evolución en 24 h (aparición de fiebre, necrosis, malestar general, ictericia, entre otros), podrían servir como criterios clínico-epidemiológicos para el diagnóstico. Al sistematizar estas variables, podrían alcanzar una alta sensibilidad y especificidad, permitiendo así un manejo precoz y eficiente del accidente. Debe considerarse el realizar un estudio para probar su validez y evaluar su eficacia, y así contribuir a controlar este problema de salud pública.

\section{Referencias bibliograficas}

1.- Maguiña C, Hinojosa J C, Gutiérrez R, Henríquez C, Ugarte C. Enfermedades por artrópodos. Parte I: Loxoscelismo cutáneo y cutáneo-visceral en el Perú. Dermatol Perú. 2004; 14: 134-9.

2.- Manríquez J, Silva S. Loxoscelismo cutáneo y cutáneovisceral: Revisión sistemática. Rev Chilena Infectol 2009; 26: 420-32.

3.- Sanabria H, Zavaleta A. Panorama epidemiológico del loxoscelismo en el Perú. Rev Peru Med Exp Salud Pública. 1997; 14: 33-41.

4.- Ríos J C, Pérez M, Sánchez P, Bettini M, Mieres J J, Paris E. Caracterización clínico-epidemiológica telefónica de la mordedura por araña de rincón, en un centro de información toxicológica de Chile, durante el año 2005. Rev Med Chile 2007; 135: 1160-5.

5.- Valverde J. Aspectos clínicos y epidemiológicos del loxocelismo, Hospital Regional Docente de Trujillo, enero 2001 a noviembre 2003. Folia Dermatol Peru 2003; 14: $15-9$.

6.- Webb C, Maguiña C, González E. Factores asociados a la presentación víscero-hemolítica de loxoscelismo en el Hospital Nacional Cayetano Heredia, entre el 2000 y 2008. Acta Méd. Peruana 2010; 27: 29-36.

7.- Mendoza C A, Cabezas C. Loxoscelismo: Evaluación clínica, tratamiento y prevención. Fecha acceso: 6 de mayo de 2013. Disponible en:http://sisbib.unmsm.edu.pe/ bvrevistas/speit/2006_n1/pdf/a02.pdf

8.- Ríos J C, Paris E, Apt W, Ristori L, Ramírez G. Guía clínica para el manejo de la mordedura de araña de rincón. Loxosceles laeta. Fecha de acceso: 20 de abril de 2006. Disponible en: www.cituc.cl/LoxoscelesLaeta.pdf.

9.- Ministerio de Salud de Chile. Guía clínica para el manejo y tratamiento de mordedura de araña de rincón (Loxosceles laeta). Santiago de Chile; 2004.

Rafael Pichardo-Rodríguez Instituto de Investigación en Ciencias Biomédicas, Universidad Ricardo Palma. Lima, Perú. Correspondencia $a$ : rafael_martin1352@hotmail.com 